\title{
Three Pillar Information Management System for Modeling the Environment of Autonomous Systems
}

\author{
Ioana Gheţa $^{1}$, Marcus Baum ${ }^{2}$, Andrey Belkin ${ }^{1}$, Jürgen Beyerer ${ }^{1,3}$, Uwe D. Hanebeck ${ }^{2}$ \\ ${ }^{1}$ Karlsruhe Institute of Technology (KIT), Institute for Anthropomatics, Vision and Fusion Laboratory \\ ${ }^{2}$ Karlsruhe Institute of Technology (KIT), Institute for Anthropomatics, Intelligent Sensor-Actuator-Systems Laboratory \\ ${ }^{3}$ Fraunhofer Institute of Optronics, System Technologies and Image Exploitation IOSB \\ Karlsruhe, Germany \\ Email:\{ioana.gheta, marcus.baum, andrey.belkin\}@kit.edu, juergen.beyerer@iosb.fraunhofer.de, uwe.hanebeck@ieee.org
}

\begin{abstract}
This contribution is about an information management and storage system for modeling the environment of autonomous systems. The three pillars of the system consist of prior knowledge, environment model and sensory information. The main pillar is the environment model, which supplies the autonomous system with relevant information about its current environment. For this purpose, an abstract representation of the real world is created, where instances with attributes and relations serve as virtual substitutes of entities (persons and objects) of the real world. The environment model is created based on sensory information about the real world. The gathered sensory information is typically uncertain in a stochastic sense and is represented in the environment model by means of Degreeof-Belief (DoB) distributions. The prior knowledge contains all relevant background knowledge (e.g., concepts organized in ontologies) for creating and maintaining the environment model. The concept of the three pillar information system has previously been published. Therefore this contribution focuses on further central properties of the system. Furthermore, the development status and possible applications as well as evaluation scenarios are discussed.
\end{abstract}

\section{INTRODUCTION}

An intelligent autonomous systems has to gather information about its environment in order to deduce adequate actions that solve a particular task. For autonomous systems acting in a complex real-world environment, such as humanoid robots helping with domestic applications, the mere storage and retrieval of information is not enough. Because such autonomous systems have to deal with uncertain and incomplete information about their relevant environment, elaborate information management mechanisms are additionally necessary.

This paper proposes an intelligent, universal information management system, whose basic concept was already introduced in [1]: the three pillar information system. As reflected by its name, the three pillar information system consists of three pillars: prior knowledge, environment model, and sensory information. The prior knowledge and the environment model pillars can be compared to the long and short term memories of an autonomous system. The environment model, which is the central component of the system, can be seen as the abstract representation of the real world. It represents the entities of the real world objects, and persons by means of instances. Sensory information, which is the interface to the real world, is employed for creating the environment model.
This contribution presents further details of the three pillar information storage and management system, e. g., information representation on abstraction levels. Furthermore, possible applications and evaluation scenarios for the system are discussed. For this purpose, an evaluation platform is introduced, which employs techniques from extended range telepresence [2]-[4]. This platform allows a human to act and cooperate with a humanoid robot in a virtual environment in a realistic manner.

a) Related Work: Modeling the environment of autonomous systems involves modeling and representing information. Common methods for doing so are based on semantic nets, predicate logic, or formal languages [5]. Nowadays, proposed methods involve ontologies, object-oriented, and probabilistic approaches [6]-[10]. Combinations of two or of all three approaches have also been proposed in the literature. Most approaches dealing with modeling the environment and representing information are domain-specific and not extendable to other applications. [11] proposes an object-oriented world modeling approach with the purpose of creating virtual environments for simulation or system engineering in the financial domain. [10] proposes a dynamic object-oriented approach for modeling the relevant environment of cooperative vehicles, incorporating attribute uncertainties. Neither of these contributions deals with the possibility of extending the model to other applications.

\section{The Three Pillar Information System}

The basic structure of the three pillar information system is given in Figure 1. The first pillar is the prior knowledge, which comprises all background information about the environment of the autonomous system. There are two types of prior knowledge. The first one is the instance-related knowledge, e. g., instances of persons or objects, maps. The second type consists of concepts formalized as ontologies, i.e., information about instances with attributes, relations, and rules. The concept-based information is context-specific and can be loaded/unloaded as needed for the autonomous system in order to prevent overfitting.

The main component of the three pillar information system is the environment model, which offers an abstract picture of the current real environment of the autonomous system. 


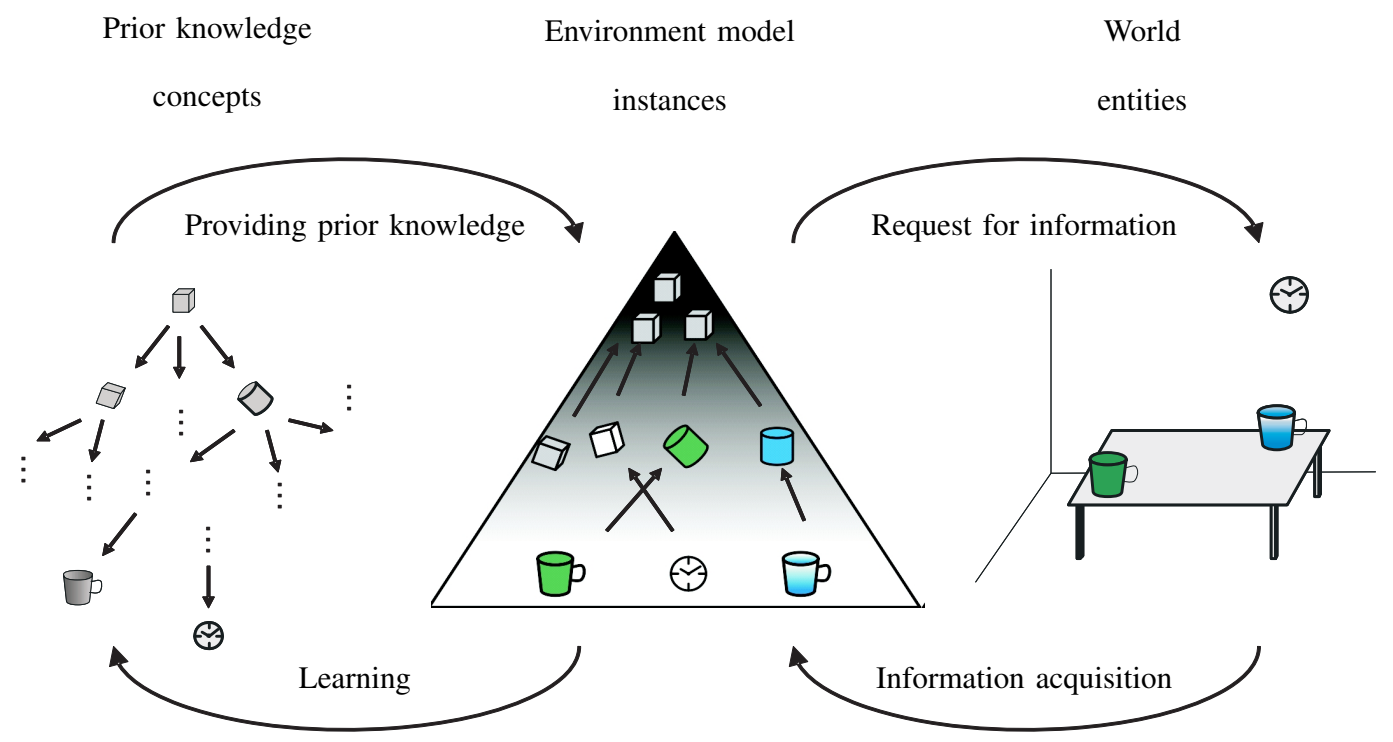

Fig. 1: Three pillar information system.

It serves as an information hub for sensors and inference processes [9], [12], [13]. In the environment model, instances with their attributes are represented as placeholders for entities of the real world. The relations between instances are modeled by means of multiple semantic networks.

The third component reflects the real world, which consists of entities like objects and persons with their attributes and relations. Observed entities (sensory information) are mapped to instances with attributes and relations in the environment model. This data association problem has been addressed in detail in [1].

\section{A. Environment Model}

In the following, the basic ideas of the probabilistic formalization of the environment model is introduced, see also [1]. As already mentioned, the environment model mainly consists of a set of instances. The number of instances at time step $k$ is denoted in the following with $N_{k}$. An instance $i$ at time step $k$ is modeled as a DoB distribution

$$
p\left(e_{k}^{i}, \underline{a}_{k}^{i}\right),
$$

where $e_{k}^{i} \in\{0,1\}$ specifies whether the instance $i$ exists $\left(e_{k}^{i}=\right.$ $1)$, and

$$
\underline{a}_{k}^{i}:=\left[\begin{array}{llll}
t_{k}^{i} & \underline{a}_{k}^{i, 1} & \cdots & \underline{a}_{k}^{i, n_{a}}
\end{array}\right]^{\mathrm{T}}
$$

is a vector that is composed of the discrete type $t_{k}^{i}$ of the instance $i$, and $n_{a}$ (discrete or continuous) other descriptive attributes $\underline{a}_{k}^{i, 1}, \ldots, \underline{a}_{k}^{i, n_{a}}$. Note that the attributes of different instances are assumed to be independent. Relations between instances are modeled as binary variables and are treated similar to attributes. For simplicity, in the following parts of the contribution the ideas are only presented for attributes. In the case of relations the representations and management mechanisms are similar.

\section{B. Information Exchange between the Three Components}

The three components of the proposed information storage and management system are connected by means of information exchange mechanisms. Figure 1 shows the main information flows according to their purpose. These mainly deliver and receive information from the environment model, as this is the ultimate information fusion target.

a) Information Exchange between the Environment Model and Prior Knowledge: Ontology information can be employed to supplement the environment model (i. e., adding new instances and/or attributes and relations) or to specify attributes and relations of already existing instances. A main challenge is the specification of the attribute type, which is generally equivalent to classification. The rules of ontologies are used for performing validity, relevance, and consistency checks [9]. Instance-related knowledge is mainly used for increases the efficiency, since the instances in prior knowledge have most attributes already specified. This information flow is represented in Figure 1 by the arrow: providing prior knowledge.

In the opposite direction, the information flow is represented in Figure 1 by the arrow: learning. This is equivalent to the process of learning new concepts or just new attributes and relations of already known concepts. It also means long term saving of meaningful instances, e. g., persons that often interact with the autonomous system. The main challenge here is the development of an efficient learning strategy.

b) Information Exchange between the Environment Model and the World: The information flow from the real world into the environment model is represented in Figure 1 by the arrow: information acquisition. The process mainly consists of initialization, update and deletion of instances (based on the existence attribute), see Section II-C. Therein, the main challenge is data association, i. e., the mapping of an 
observation to an instance [1].

In the opposite direction, the information exchange process is equivalent to an information request shown in Figure 1 by the arrow with the same name. The purpose of information requests is filling the gaps (only possible when prior knowledge is incorporated) or (re)confirmation of information (e.g., for lowering the uncertainty). In this case, the challenge consists in developing high-level inference mechanisms.

\section{Information Management}

In the following, the information management process in the environment model is described. For this purpose, a mechanism for initiating and deletion of new instances is required. Moreover, the environment model has to be kept up-to-date by means of incorporating new information from sensors and predicting the state of the environment to the next time step.

1) Observation Model for Information: The environment model is updated over time by means of sensory information. For this purpose, an observation model that relates the given observation to the attributes of an instance is required [1].

We assume that at each time step $k$, a single observation is received from a sensor. This observation is assumed to regard a single (unknown) instance. The instance an observation regards, is modeled with an association variable $d_{k} \in\left\{0,1 \ldots, N_{k}\right\}$. The probability that the observation at time step $k$ regards the instance $d_{k}$ is given by

$$
p\left(d_{k} \mid \underline{e}_{k}\right)
$$

with $\underline{e}_{k}:=\left[e_{k}^{1}, \ldots, e_{k}^{N_{k}}\right]^{\mathrm{T}}$ and $N_{k}$ as the number of instances in the environment model. An observation that regards a new instance, is indicated by $d_{k}=0$.

The instance observation model specifies the probability of an observation, if it is known to regard instance $i$ :

$$
\begin{aligned}
p\left(\underline{y}_{k}^{i} \mid \underline{a}_{k}^{i, 1}, \ldots \underline{a}_{k}^{i, n(i)}, t_{k}^{i}, e_{k}^{i}, d_{k}=i\right):= \\
p\left(\underline{y}_{k}^{i, 1} \mid \underline{a}_{k}^{i, m_{1}}\right) \cdot \ldots \cdot p\left(\underline{y}_{k}^{i, r} \mid \underline{a}_{k}^{i, m_{r}}\right) \cdot p\left(\mathcal{M}_{k} \mid t_{k}^{i}\right) .
\end{aligned}
$$

The observation vector $\underline{y}_{k}^{i}:=\left[\underline{y}_{k}^{i, 1}, \ldots, \underline{y}_{k}^{i, r}, \mathcal{M}_{k}\right]^{\mathrm{T}}$ consists of individual observations of particular attributes $\underline{y}_{k}^{i, 1}, \ldots, \underline{y}_{k}^{i, r}$ and an index set that gives the measured attributes, i. e., $\mathcal{M}_{k}^{-}=$ $\bigcup_{s} m_{s}$. The observation model for a single attribute is

$$
p\left(\underline{y}_{k}^{i, s} \mid \underline{a}_{k}^{i, m_{s}}\right),
$$

where $m_{s}$ denotes the attribute observed by $\underline{y}_{k}^{i, s}$. The set of observed attributes depends on the particular type of the instance, e.g., for a plate it is likely to measure color, position and form. For this purpose, the conditional $\operatorname{DoB} p\left(\mathcal{M}_{k} \mid t_{k}^{i}\right)$ specifies the DoB of observing the attributes $\mathcal{M}_{k}$, when the type of the instance is given.

Finally, an observation model for new unknown instances, i. e., that have not been observed so far is employed. It is given by

$$
p\left(\underline{y}_{k}^{i} \mid \underline{a}_{k}^{i, 1}, \ldots \underline{a}_{k}^{i, n(i)}, t_{k}^{i}, e_{k}^{i}=1, d_{k}=i\right) .
$$

Thus, this model allows for expressing that a new instance representing the given entity may have a specific attribute value. For example, this may model that the entity is more probable to be situated on a table than on the floor.

2) Dynamic Model for Information: The relevant environment of the autonomous system is subject to changes as time passes. In order to capture the dynamic evolution, a dynamic model for information as presented in the following is required.

The existence probability for an instance is assumed to decrease over time according to the Markov model

$$
\begin{aligned}
& p\left(e_{k}^{i}=1 \mid e_{k-1}^{i}=1\right)=\beta^{i}, \\
& p\left(e_{k}^{i}=1 \mid e_{k-1}^{i}=0\right)=0,
\end{aligned}
$$

where $0<\beta^{i} \leq 1$ is a constant, see Section II-C4. Hence, a disappeared instance does not re-appear.

The temporal evolution of descriptive attributes is modeled with a conditional DoB of the form

$$
p\left(\underline{a}_{k+1}^{i} \mid \underline{a}_{k}^{i}\right) .
$$

An example for a simple temporal evolution model for a continuous attribute $\underline{a}_{k}^{i}$ could be

$$
p\left(\underline{a}_{k+1}^{i} \mid \underline{a}_{k}^{i}\right)=\mathcal{N}\left(\underline{a}_{k+1}^{i}-\underline{a}_{k}^{i}, \mathbf{C}_{k}^{v}\right),
$$

which results from the motion equation

$$
\underline{a}_{k+1}^{i}=\underline{a}_{k}^{i}+\underline{v}_{k},
$$

where $\underline{v}_{k}$ denotes additive Gaussian system noise with covariance matrix $\mathbf{C}_{k}^{v}$. This dynamic model increases the uncertainty for $\underline{a}_{k}^{i}$ in order to capture small changes.

3) Initialization and Deletion of Instances: Usually, the exact number of instances in the real world is unknown. For example, a humanoid robot is not aware of the exact number of cups in the kitchen. Hence, a mechanism that decides when to trigger tracking a new instance is required.

If the probability that the observation $\underline{\hat{y}}_{k}$ regards a new instance exceeds a threshold $\gamma_{\mathrm{e}}$

$$
p\left(d_{k}=0 \mid e_{k}^{0}=1, \underline{\hat{y}}_{1: k}\right)>\gamma_{\mathrm{e}},
$$

the observation is assumed to regard a new instance. Hence, in addition to the already existing instances

$$
p\left(e_{k}^{1}, \underline{a}_{k}^{1}\right), \ldots, p\left(e_{k}^{N_{k}}, \underline{a}_{k}^{N_{k}}\right),
$$

a new possible instance

$$
p\left(e_{k}^{N_{k}+1}, \underline{a}_{k}^{N_{k}+1}\right)
$$

is initialized with a suitable prior DoB for its existence and attributes. However, the existence of the instance has to be confirmed with the help of subsequent measurements. If the probability of the existence of the instance exceeds an instantiation threshold

$$
p\left(e_{k}^{i}=1\right)>\gamma_{\mathrm{i}}
$$

it is considered to exist. 
In the same manner, an instance is deleted from the environment model, if the DoB of its existence drops below the deletion threshold

$$
p\left(e_{k}^{i}=1\right)<\gamma_{\mathrm{e}},
$$

with $\gamma_{\mathrm{e}}<\gamma_{\mathrm{i}}$. The relation between $\gamma_{\mathrm{e}}$ and $\gamma_{\mathrm{i}}$ is necessary for ensuring a hysteresis, i.e., a created instance should not be immediately deleted, see Figure 2.

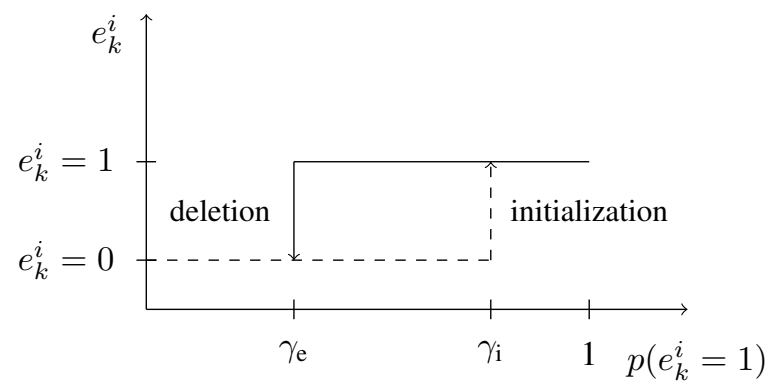

Fig. 2: Hysteresis for the instantiation and the deletion of instances.

Figure 3 shows an example of the life-cycle of an instance by means of the DoB for its existence attribute. Starting with its initialization at time step $u=5$, when the DoB higher is than the initialization threshold, the continuous blue line shows the life cycle, when the existence of the instance is reconfirmed at the time steps $k=6$ and $v=10.5$. When no new information is acquired, the DoB may drop under the deletion threshold at several time steps as shown by the red dashed line.

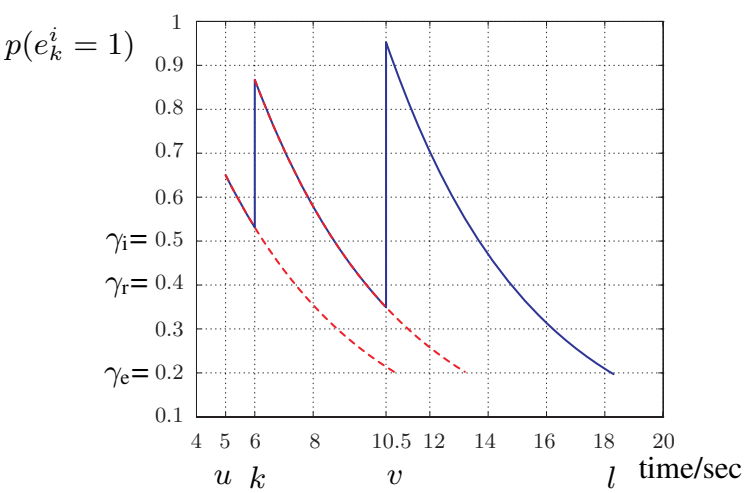

Fig. 3: Example of the life-cycle of an instance. The red dashed line describes the propagation resulting from the aging mechanism, if no new observation is available. The continuous line describes the course of the DoBs when the existence of the entity is reconfirmed at the time steps $k=6$ and $v=10.5$.

4) Time Propagation and Observation Update of the Environment Model: This section outlines the basic mechanism for performing a Bayesian update of the environment model based on the observation received from sensors. Furthermore, the state of the environment has to be propagated to the next time by means of a dynamic model.
The aim is to recursively compute the existence probabilities and the DoB distributions of descriptive attributes for all instances $i$

$$
p\left(e_{k}^{i}=1 \mid \underline{\hat{y}}_{1: k}\right) \quad \text { and } \quad p\left(\underline{a}_{k}^{i} \mid e_{k}^{i}=1, \underline{\hat{y}}_{1: k}\right) .
$$

For this purpose, the common prediction/filtering scheme of Bayesian state estimation is employed.

The prediction step takes the state of the environment model at time step $k-1$ and propagates it to the next time step $k$. The result is the predicted existence and the predicted attributes

$$
p\left(e_{k}^{i}=1 \mid \underline{\hat{y}}_{1: k-1}\right) \quad \text { and } \quad p\left(\underline{a}_{k}^{i} \mid e_{k}^{i}=1, \underline{\hat{y}}_{1: k-1}\right) .
$$

for all instances $i$.

At each time step $k$, a single observation $\hat{\hat{y}}_{k}$ is received. The Bayesian filtering step (observation update step) takes the prediction (6) and $\hat{y}_{k}$ in order to computed the updated state (5). The major challenge with the filtering step is that it is not known, which instance the observation regards. This problem including the derivation of the mathematical foundations has been discussed in detail in [1]

\section{Abstraction Levels of Information}

The information in the environment model is structured on different abstraction levels depending on the degree of detail expressed by attributes and relations. An instance having a high number of specified attributes and relations is situated on a low abstraction level. An instance having only the existence attribute specified (i.e., no descriptive attributes have been observed) is situated on the highest abstraction level. On the lowest abstraction level, instances are situated for which all attributes and relations are specified. An instance may change to a lower abstraction level, when new information is available such that more of its attributes and relations are specified. The abstraction levels are presented in Figure 4.

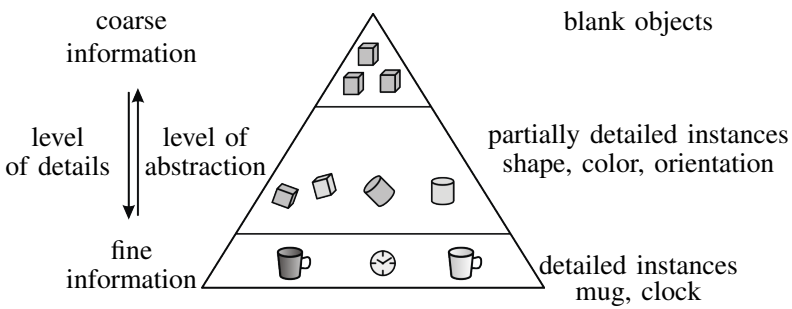

Fig. 4: Abstraction levels in the environment model.

The abstraction levels are also very useful when retrieving information. Depending on the task, different degrees of detail of the retrieved information may be necessary. As an example, for automatic path planning it is sufficient to know the free space in the environment, i.e., only information on a high abstraction level is required, e.g., only the attributes position and extension of the instances. On the other hand, for a grasp task detailed information is required, e. g., attributes like $3 D$ shape or grasp possibility and relations like is situated on of the respected instance are necessary. This information is situated on a low abstraction level. 


\section{State of the ReAlization}

The three pillar information architecture is currently realized for a a humanoid robot helping with domestic applications.

Until now, the framework for the middle component (the environment model) is implemented. This includes the following:

- instances are saved and serve as containers for attributes;

- attributes may be expressed as Gaussian mixture distributions;

- relations between instances are saved in form of graphs by means of multiple semantic networks.

Moreover, the basic mechanisms for the information exchange between the world and the environment model are implemented. Further on, the basic mechanisms for exchanging information with prior knowledge are also provided. Currently, only an ontology with a small number of concepts is being employed. In the future, the use of complete ontologies is intended. Some of the predefined concepts in the prior knowledge (e. g., cups, bottles, laptops) with their properties (e.g., 3D point clouds, textures) were obtained by employing a 3D laser scan system.

The environment model is implemented as multi-threading cross-platform distributed system written in C++ with STL, Boost, and OGRE libraries. The data is represented in the XML format with serialization and network transmission possibility. The 3D visualization is performed by OGRE scene renderers for a better comparison of real world and the environment model.

\section{ApPlicATiON}

One application of the three pillar information system is within the DFG Collaborative Research Center (Sonderforschungsbereich) SFB 588 "Humanoid Robots-Learning and Cooperating Multimodal Robots". ${ }^{1}$ The purpose of the project is to build a humanoid robot [14], [15] helping in the household. For development and testing purposes a kitchen environment has been created. There, the humanoid robot can be tested when performing tasks of different difficulties and different contexts, e.g., helping with cooking, operating kitchen appliances (like dish washer or fridge), setting the table. Details of development and implementation as well as practical examples are given in [12], [13].

In this context, a test environment for human-robot cooperation based on a extended range telepresence system [2][4] has been developed. In this test environment, the user can cooperate with a virtual humanoid robot in a virtual household environment. The interaction with the virtual environment is enabled by a data glove and a head-mounted display. A technique called motion compression [2], [3] allows walking in a large virtual environment, e. g., a household, even if the user environment is small. The extended range telepresence system allows realistic experiments and focusing on the actual

\footnotetext{
${ }^{1}$ http://www.sfb588.uni-karlsruhe.de
}

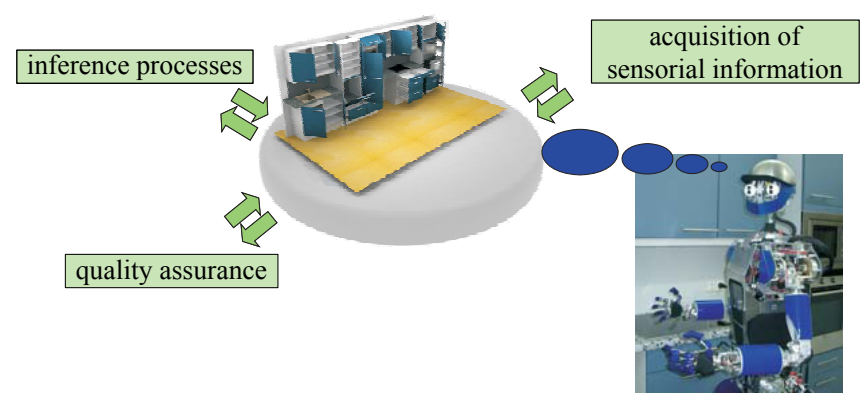

Fig. 5: Environment model for a humanoid robot.

interaction without physical or technical restrictions. Furthermore, experiments in the virtual environment are repeatable under exactly the same conditions.

One possible human-robot cooperation scenario in the virtual environment is the construction of a shelf together with a humanoid robot (see Figure 6). For this purpose, it is necessary to stick together boards by means of dowels. The humanoid robot is supposed to assist the user and it should always be on the spot whenever it can help. Therefore, the robot is able to autonomously initiate supporting actions [16]. Furthermore, the virtual humanoid robot is able to enact different roles for assisting the user. For instance, the robot can bring dowels or hold a board. The goal is to allow humans to work with the robot in the same way as they are used to cooperate with other humans. As a personal assistant, the robot has to adapt its behavior to the special needs of the human at anytime. In order to deduce the needs of the human and to perform actions, the robot must have enough information about the objects in its nearby. For instance, the robot should know, where the dowels are and what action the user is performing. For this purpose, the introduced three pillar information system could be employed.

It is interesting to note that the extended range telepresence system also offers the possibility for Wizard of Oz experiments [17] with the real humanoid robot. Namely, the telepresence system can be used by a human to control a real humanoid robot in an intuitive manner. The humanoid robot can then cooperate with a human, who is not aware that the robot is in fact controlled by human. These kind of experiments help to derive behavior rules for a humanoid robot.

\section{COnClusions And Future Work}

This work has given a detailed introduction and overview of a universal information storage and management system for modeling the environment of autonomous systems, called the three pillar information system. The three main components of the system are prior knowledge, environment model, and sensory information. The main component is the environment model, which supplies the autonomous system with information about its environment. The environment model maintains an abstract representation of the real world. It consists of instances with attributes and relations that serve as virtual substitutes of entities (persons and objects) of the real world. The environment model has been formulated within Baysian 


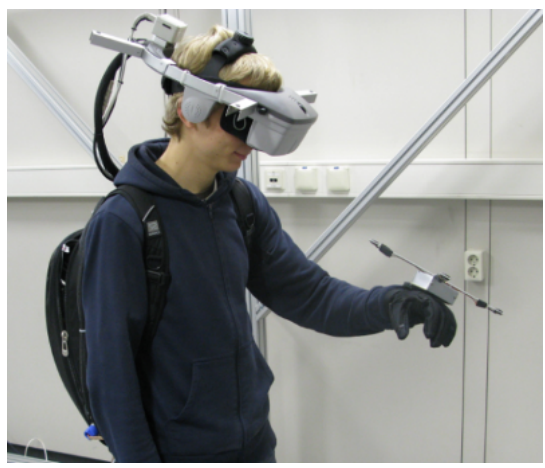

(a) User environment of the telepresence system.

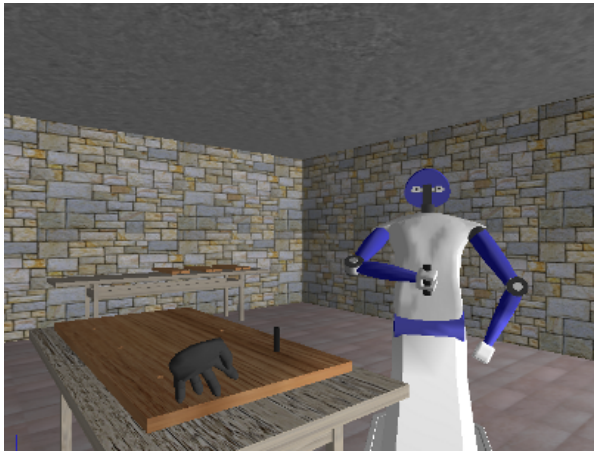

(b) Screenshot of the user's view in the virtual environment. The virtual humanoid robot brings the user dowels.

Fig. 6: Extended range telepresence system for the evaluation of human-robot-interaction.

probability theory in order to deal with stochastic uncertainties. Based on the Bayesian framework, decisions regarding the initialization, update, and deletion of information are made. Besides the probabilistic approach, another important feature of the system is the representation of information on different abstraction levels. According to the degree-of-detail based on the number of specified attributes and relations, an instance may be situated on different abstraction levels. Thus, retrieval of information according to given tasks is simplified. The basic functions of the system have already been developed. These include storing and representing information and some basic check mechanisms, e. g., validity.

Finally, applications of the three pillar information storage and management system are discussed. One of these is a humanoid robot helping with domestic applications. An extended range telepresence system can be employed to evaluate the proposed three pillar information system. This system allows for interacting with a virtual humanoid robot in a virtual environment in order to solve tasks, e. g., a shelf construction.

Future work will be concerned with developing strategies for solving the remaining challenges within the information flows between the components of the three pillar information system. An example is solving the classification problem, i. e., mapping of instances from the environment model to concepts from prior knowledge. The challenges involve the integration of semantic knowledge, e.g., about attributes and the search for appropriate methods for comparing DoB distributions. Moreover, a detailed evaluation including experiments of the approach is left for future work.

\section{REFERENCES}

[1] M. Baum, I. Gheta, A. Belkin, J. Beyerer, and U. D. Hanebeck, "Data Association in a World Model for Autonomous Systems," in Proceedings of the 2010 IEEE International Conference on Multisensor Fusion and Integration for Intelligent Systems (MFI 2010), Salt Lake City, Utah, Sep. 2010.

[2] N. Nitzsche, U. D. Hanebeck, and G. Schmidt, "Motion Compression for Telepresent Walking in Large Target Environments," Presence: Teleoperators \& Virtual Environments, vol. 13, no. 1, pp. 44-60, Feb. 2004.
[3] P. Rößler, U. D. Hanebeck, and N. Nitzsche, "Feedback Controlled Motion Compression for Extended Range Telepresence," in Proceedings of the 2004 IEEE International Conference on Mechatronics \& Robotics (MechRob 2004), Aachen, Germany, Sep. 2004, pp. 1447-1452.

[4] O. C. Schrempf, D. Albrecht, and U. D. Hanebeck, "Tractable Probabilistic Models for Intention Recognition Based on Expert Knowledge," pp. 1429-1434, Nov. 2007

[5] P. Russell, Stuart J. ; Norvig, Artificial intelligence, 3rd ed. Pearson Education, 2010.

[6] A. Bauer, "Probabilistic Reasoning on Object Occurrence in Complex Scenes," in Image and Signal Processing for Remote Sensing XV, Proc. of SPIE, vol. 7477, 2009.

[7] P. C. G. da Costa, K. B. Laskey, and K. J. Laskey, "PR-OWL: A Bayesian Ontology Language for the Semantic Web," in ISWC-URSW, 2005, pp. 23-33.

[8] W. V. Siricharoen, "Ontologies and Object models in Object Oriented Software Engineering," IAENG International Journal of Computer Science, vol. 33, no. 1, 2007.

[9] I. Gheța, M. Heizmann, and J. Beyerer, "Object Oriented Environment Model for Autonomous Systems," in Proceedings of the second Skövde Workshop on Information Fusion Topics, H. Boström, R. Johansson, and J. van Laere, Eds. Skövde Studies in Informatics, Nov. 2008, pp. 9-12.

[10] Z. Papp, C. Brown, and C. Bartels, "World Modeling for Cooperative Intelligent Vehicles," in IEEE Intelligent Vehicles Symposium, 2008, pp. 1050-1055.

[11] S. Isoda, "Object-Oriented World-Modeling Revisited," Journal of Systems and Software, vol. 59, no. 2, pp. 153-162, 2001.

[12] A. Belkin, "Object-Oriented World Modeling for Autonomous Systems," Karlsruhe Institute of Technology KIT, Tech. Rep., 2010.

[13] B. Kühn, A. Belkin, A. Swerdlow, T. Machmer, J. Beyerer, and K. Kroschel, "Knowledge-Driven Opto-Acoustic Scene Analysis based on an Object-Oriented World Modelling Approach for Humanoid Robots," in Proceedings of the 41st International Symposium on Robotics and the 6th German Conference on Robotics. VDE-Verlag, 2010.

[14] T. Asfour, K. Regenstein, P. Azad, J. Schroder, A. Bierbaum, N. Vahrenkamp, and R. Dillmann, "ARMAR-III: An integrated humanoid platform for sensory-motor control," in 6th IEEE-RAS International Conference on Humanoid Robots, 2006, pp. 169-175.

[15] T. Asfour, P. Azad, N. Vahrenkamp, K. Regenstein, A. Bierbaum, K. Welke, J. Schröder, and R. Dillmann, "Toward Humanoid Manipulation in Human-Centred Environments," Robot. Auton. Syst., vol. 56, no. 1 , pp. 54-65, 2008.

[16] O. C. Schrempf, U. D. Hanebeck, A. J. Schmid., and H. Wörn, "A Novel Approach to Proactive Human-Robot Cooperation," in Proceedings of the 2005 IEEE International Workshop on Robot and Human Interactive Communication (ROMAN 2005), Nashville, Tennessee, Aug. 2005, pp. 555-560.

[17] L. Molin, "Wizard-of-Oz Prototyping for Co-Operative Interaction Design of Graphical User Interfaces," in NordiCHI '04: Proceedings of the third Nordic conference on Human-computer interaction. New York, NY, USA: ACM, 2004, pp. 425-428. 